\title{
Applications in Molecular Ultrasound Imaging: Present and Future
}

\author{
Vishal Thumar, MD ${ }^{a}$, Ji-Bin Liu, MD ${ }^{b}$, John Eisenbrey, PhD ${ }^{b, *}$ \\ ${ }^{a}$ Department of Internal Medicine, Lankenau Medical Center, Wynnewood, PA, USA; ${ }^{b}$ Department of Radiology, Thomas Jefferson \\ University, Philadelphia, PA, USA \\ Received May 7, 2019; revision received July 1; accepted July 6.
}

\begin{abstract}
Molecular ultrasound imaging or targeted contrast-enhanced ultrasound (CEUS) is a relatively new technique that has varied applications to augment both diagnostics and therapeutics. Ultrasound contrast agents are conjugated to ligands that bind with specific biomarkers in the areas of interest which can then be quantified using ultrasound technology. This technique has numerous clinical applications including studying pathophysiology of disease, improving diagnostic sensitivity and specificity, and improving localized drug delivery. This technology, most notably, has proven useful in numerous oncologic and cardiovascular applications. Given ultrasound's advantages over other radiographic studies including its low cost, lack of ionizing radiation, portability, ability to provide real-time imaging, and non-invasiveness, recent investigations have expanded the utility of molecular ultrasound. In this review, we briefly review targeted ultrasound contrast agents and explore the current applications of molecular ultrasound as well as future applications based on the currently published literature.
\end{abstract}

Key words: Molecular ultrasound imaging; Contrast-enhanced ultrasound; Therapeutics; Contrast media

Advanced Ultrasound in Diagnosis and Therapy 2019;03:062-075

DOI: 10.37015/AUDT.2019.190812

$\mathrm{M}$ olecular ultrasound imaging or targeted contrast-enhanced ultrasound (CEUS) is a relatively novel technique that combines the use of ultrasound contrast agents conjugated to ligands that bind with specific biomarkers in the areas of interest. These agents can accumulate at their target passively (attachment of the shell of the contrast agent to cells) or actively (modification to the shell to allow attachment to specific ligands). Once administered, the contrast agent with the specific ligand accumulates in the area with the highest affinity to the target molecule (small proteins, antibodies, etc.). Ultrasound technology allows quantitative detection of expression of the target molecule which allows a wide scope of diagnostic and therapeutic applications of molecular ultrasound. The promising utility of targeted CEUS combined with the relative convenience of using ultrasound including its low cost, lack of ionizing radiation, portability, real-time imaging, and non-invasiveness, makes this technology ideal to also explore the pathophysiology of certain diseases, particularly in the cardiovascular field. The most beneficial application of CEUS and nonnephrotoxic ultrasound contrast agents is when computed tomography $(\mathrm{CT})$ and magnetic resonance imaging (MRI) are contraindicated $[1,2]$.

The contrast agents used for targeted CEUS can be divided into two main categories, microbubble and non-microbubble, each of which have its own unique applications. Microbubbles consist of a gas core encased by a hard shell which can be composed of various materials including sugars, proteins, polymers, and lipids (Fig. 1). The shell prevents gas diffusion and, by adding a polyethylene glycol component, reduces aggregation. Different gases have been used for the core component of the microbubble, of which heavier, biologically inert gases are more stable and circulate longer intravascularly. Microbubbles are typically 1 to $6 \mu \mathrm{m}$ in diameter, thus usually contained within the vascular space. This makes microbubbles ideal for intravascular imaging rather than imaging extravascular components [1,2].

\footnotetext{
* Corresponding author: Department of Radiology, Thomas Jefferson University, 796 Main Building, 132 South $10^{\text {th }}$ Street, Philadelphia, PA 19107, USA

e-mail: John.Eisenbrey@jefferson.edu unrestricted use, distribution and reproduction in any medium provided that the original work is properly attributed.
} 
Non-microbubble contrast agents are generally smaller than microbubble contrast agents $(10-1000 \mathrm{~nm})$ and are therefore better at entering the extravascular space (Fig. 2). One major disadvantage of these contrast agents is the poor acoustic reflectivity of the liquid or solid colloid core due to its small size and incompressible nature, making imaging with ultrasound difficult. To optimize visualization, different formulations of these contrast agents have been synthesized. An example of this is vaporization of the liquid core of a perfluorocarbon (PFC) nanodroplet into gas to increase ultrasound detection. Non-microbubble contrast agents are particularly useful in drug delivery due to its ability to enter the extravascular space. When nanobubbles, for example, are exposed to ultrasound acoustic waves after aggregating in the target in the extravascular space, they release chemotherapeutic agents in tumors $[1,2]$.
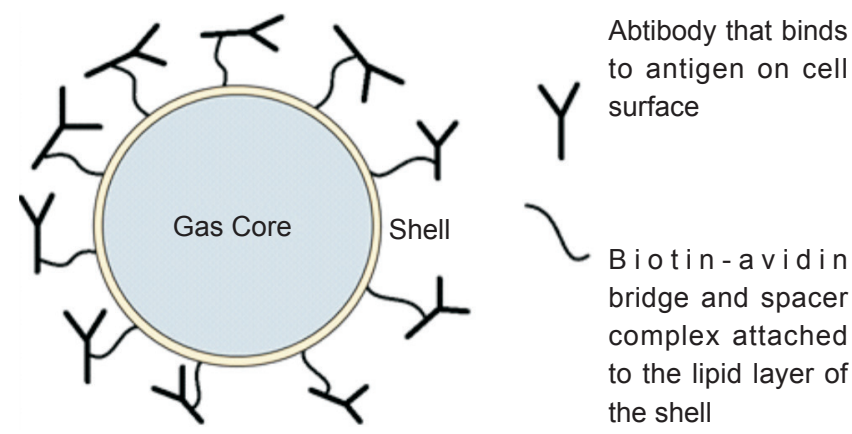

Figure 1 Illustration of a targeted microbubble complex composed of microbubble with gas core linked to antibody by a biotin-avidin and spacer complex.

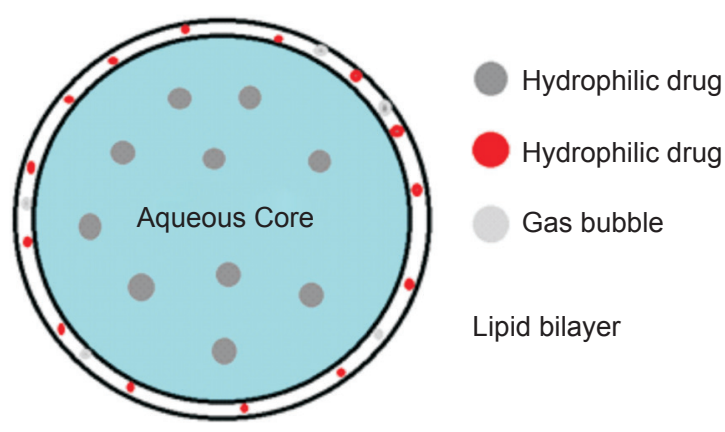

Figure 2 Illustration of a drug-loaded liposome with an aqueous core and gas bubbles in the lipid bilayer (can also be confined within the inner lipid layer in the same component as the aqueous core). Hydrophilic drugs can be loaded within the aqueous core and hydrophobic drugs can be loaded within the lipid bilayer.

There are multiple recent literature reviews discussing contrast agents. Chong's review on the current and future applications of ultrasound contrast agents explored contrast agents targeting molecules such as vascular endothelial growth factor receptor 2 (VEGFR2) and alpha-v beta-3 (avb3) and the first agent in human clinical trials, BR55 [3]. These targets have been utilized in many recent studies to evaluate neoplastic angiogenesis and drug or gene therapy for cancer. New advancements in technology including superharmonic ultrasound, high frame rate, and superresolution ultrasound are also discussed, all of which could potentially provide higher resolution images [3]. Guvener's review of applications of ultrasound contrast agents similarly discussed the above applications as well as recent advancements such as drug delivery through the blood-brain barrier [4]. Wang's review of microbubbles discusses the current microbubble design trend and notably reviews current microbubbles in clinical trials or clinical usage including Sonazoid which shows promise in liver cancer monitoring and image-guided procedures and BR55 which continues to be explored in detecting and monitoring malignancies in multiple organs [5]. Zlitni's concise review discusses BR55 and recent applications of nano-sized contrast agents [6]. As reviewed in these recent papers, there are multiple promising and unique applications of ultrasound contrast agents.

This review will discuss advancements in the literature of current potential human applications of molecular ultrasound, and future potential applications of molecular ultrasound as demonstrated in animal models.

\section{Oncological Applications}

\section{Breast}

There have been multiple advancements in molecular targets for breast cancer imaging. The most established contrast agent, BR55 (MDKDR, Bracco Suisse), was used in a study to explore early breast cancer detection via VEGFR2 expression. The study was conducted using breast cancer xenografts in mice and found that VEGFR2 expression decreased with tumor growth but expression was still detectable with imaging. Using BR55 targeting VEGFR2 to detect early angiogenesis is a promising modality for breast cancer detection [7]. A later firstin-human study using the BR55 targeting kinase insert domain receptor (KDR) in breast and ovarian lesions found good correlation of KDR expression with immunohistochemistry (IHC) without safety concerns about BR55, establishing the promising use of this contrast agent in targeting malignant breast and ovarian lesions [8].

Other contrast agents and molecular targets have been studied in animal models. Wilson and Bachawal explored with use of B7-H3 (CD276) as a target for breast cancer detection and found strong correlation with IHC $[9,10]$. A later study by Wilson and Bachawal found that this molecular target can detect disease status (DCIS and invasive carcinoma versus normal or hyperplastic tissue) with high sensitivity and specificity [11]. 
Multiple researchers in the past few years have created novel contrast agents conjugated to different ligands to image breast cancer in animal models. Abou-Elkacem engineered a new fibronectin scaffold ligand attached to microbubbles that targets VEGFR2 in breast cancer and found significantly increased binding to breast cancer tissue versus normal tissue. Since this novel ligand is small and lacks disulfide bonds and glycosylation, it can be cheaper and produced more efficiently [12]. Jiang engineered a new Herceptin-targeted nanobubble contrast agent (NBs-Her) for patients with HER-2 positive breast cancer. This contrast agent with Her ligand has greater half life and tumor specificity that could also potentially make it useful for therapy [13] (Fig. 3). Li created fluorescent nanobubbles conjugated to PNBL-NPY ligand to target $\mathrm{Y} 1$ receptors for breast cancer imaging [14]. Du created a perfluoropropane (C3 F8)-filled poly(lactic-coglycolic acid) (PLGA) nanobubble that targets both HER2 and VEGFR2 which may enhance ultrasound images due to its dual-targeting capability [15]. Xu explored the dual-targeting capability of this novel contrast agent further and found that dual-targeted agent had more intense signal than single-targeted agent [16]. Wischhusen created netrin-1 microbubbles as a potential diagnostic and therapeutic marker for interference therapy in breast cancer [17].

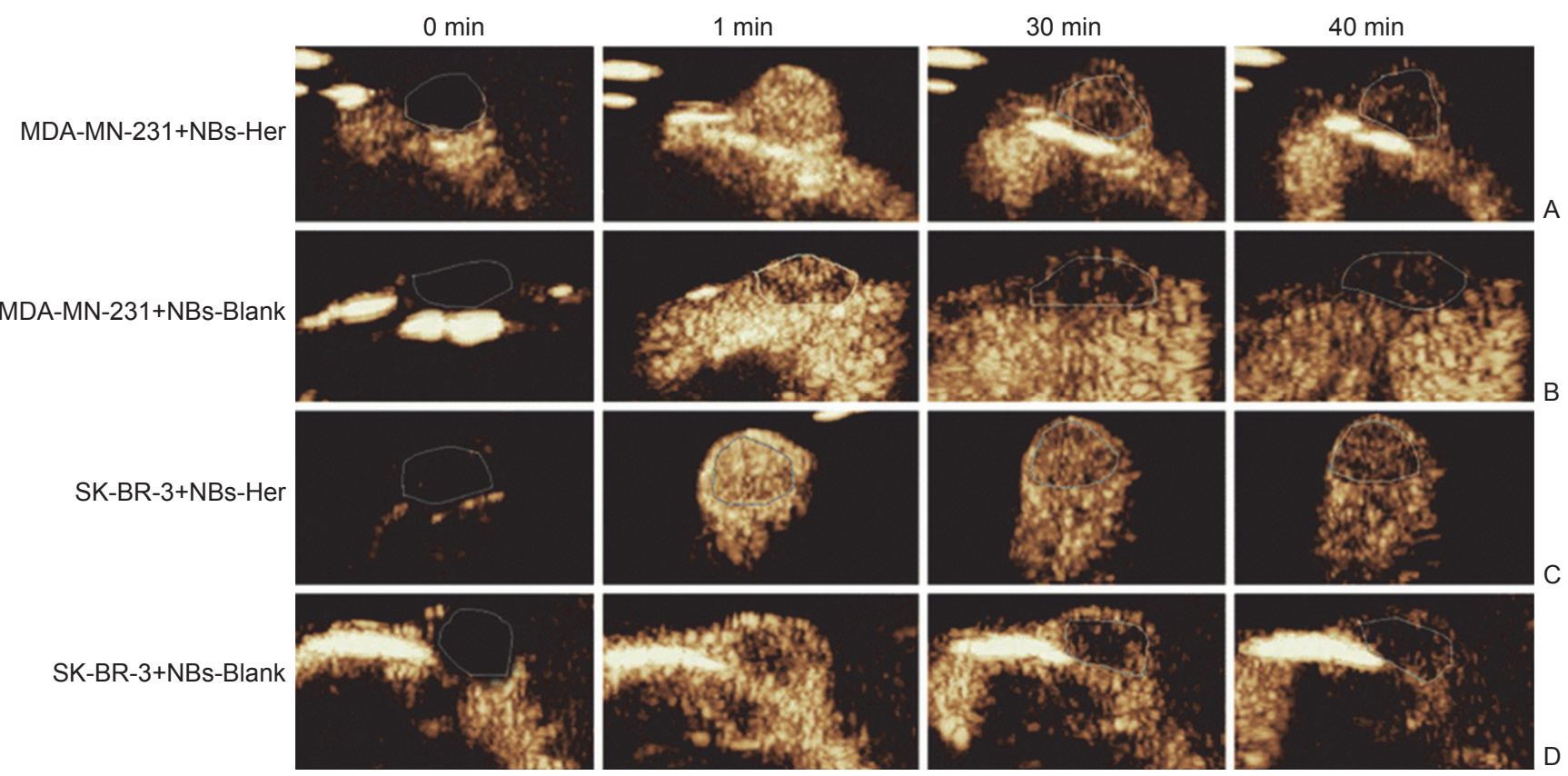

Figure 3 In vivo tumor targeting. Images were taken at the indicated time points $(0,1,30$, and $40 \mathrm{~min})$ after nanobubbles were injected into the transplanted SK-BR-3 tumors injected with Herceptin-conjugated nanobubbles (NBs-Her; A) or unconjugated nanobubbles (NBs-Blank; B) and in the transplanted MDA-MB-231 tumors injected with NBs-Her (C) or NBs-Blank (D) (Reprinted with permission from reference [13])

As seen in the above studies, there have been numerous new advancements in molecular targets and contrast agents as well as their applications for identifying breast cancer earlier and treating breast cancer more effectively. The potential clinical application of the BR55 contrast agent is promising since it has shown some success in clinical trials, but future studies can also explore further applications of this contrast agent and human application of more novel contrast agents.

\section{Prostate}

Most molecular imaging studies in prostate cancer focused on angiogenesis evaluation and new molecular targets for therapy. Early studies explored VEGFR2, intracellular adhesion molecular-1 (ICAM-1), and $\alpha \mathrm{v} \beta 3$ -integrin expression using microbubbles $[18,19]$. As discussed above, BR55 is the first contrast agent to be explored in humans. Smeenage investigated the safety and feasibility of this agent in targeting VEGFR2 and found that patients with prostate cancer had detectable levels of BR55 which can be utilized in further studies with further fine-tuning of doses to determine clinical significance [20].

A new ligand was investigated in the recent literature to detect prostate cancer which may improve current limitations with imaging specificity. Anti-prostate specific membrane antigen (PMSA) A10-3.2 aptamer was created and conjugated with nanobubbles to target and accumulate in PMSA positive cells in mice with prostate cancer xenografts [21]. In another study, porphyrin-grafted lipid (PGL) microbubbles were created 
to attempt photodynamic therapy (PDT) for prostate cancer. To improve some limitations of visualizing target lesions with conventional ultrasound, molecular ultrasound using these contrast agents improved visualization and targeting which subsequently helped administer photodynamic therapy [22] (Fig. 4 and 5).

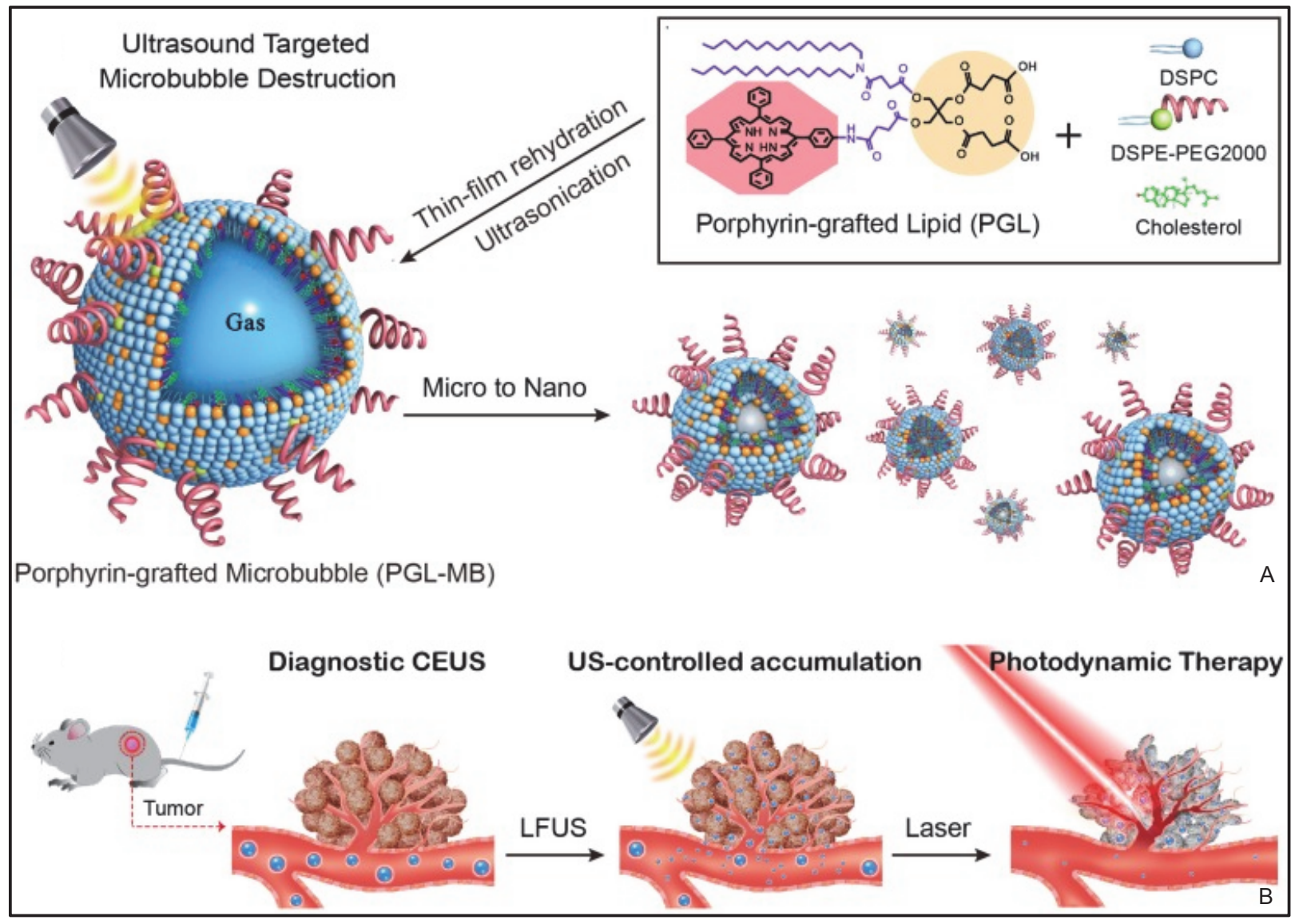

Figure 4 Schematic of the microbubble-based, ultrasound-assisted PDT strategy. (A) Preparation of PGL-MB and its transformation from microbubbles to nanoparticles under exposure to low-frequency ultrasound (LFUS); (B) Experimental process of in vivo PDT under the guidance of CEUS imaging, followed by ultrasound targeted microbubble destruction (UTMD). (Reprinted with permission from reference [22])

The utility of angiogenesis markers has been established in animal studies and new markers are now being targeted for therapy purposes. Future investigations will focus on furthering the applications of BR55, as well as other promising ultrasound contrast agents for imaging and therapy for prostate cancer.

\section{Lymph Nodes}

The utility of peri-tumoral injection of microbubbles has been established to identify sentinel lymph nodes but overall sensitivities for predicting tumor involvement have been poor. Nam et al. explored sentinel lymph nodes in metastatic melanoma using a dual-targeted molecular ultrasound agent. Microbubbles were conjugated with $\alpha v \beta 3$-integrin and P-selectin antibodies. Following peri-tumoral injection of Sonazoid in a swine melanoma model, sentinel lymph nodes were identified using lymphosonography. After clearance of Sonazoid within the node, intravascular injection of targeted microbubbles was performed to assess tumor involvement of all identified nodes. The mean intensity of the metastatic lymph nodes was significantly higher than benign nodes, thus indicating potentially new targets to detect metastatic melanoma (Fig. 6 and 7) [23].

\section{Colon}

Colon cancer is another disease where molecular ultrasound has proved beneficial, especially with the use of BR55 and molecular targets such as VEGFR2. Hackl explored detection of liver metastasis of colorectal cancer with BR55 contrast agent targeting VEGFR2 in mice. With the use of BR55, smaller lesions were more accurately detected and can be used to monitor hepatic metastasis of colon cancer [24].

More recently, studies have shown advancements in monitoring therapy and angiogenesis by targeting VEGFR2. After regorafenib therapy, tumor perfusion 
was assessed using VEGFR2 binding in one study. Under therapy, tumor perfusion and microbubble binding were reduced, showing response to therapy [25]. Similarly, two other studies using 3D-ultrasound imaging of microbubbles targeting VEGFR2 showed decreased VEGFR2 expression after treatment [26,27].

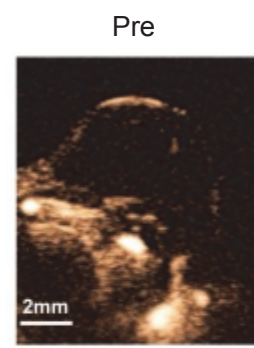

$5 \mathrm{~s}$ after injection
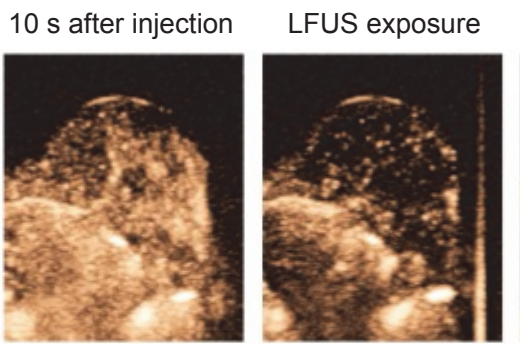

After LFUS
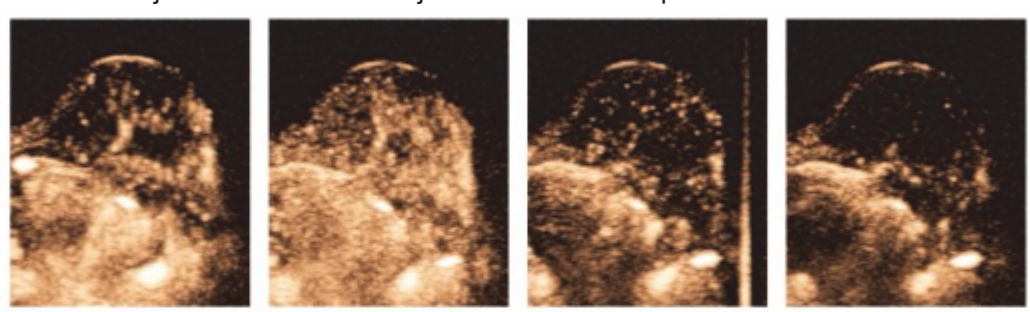

A

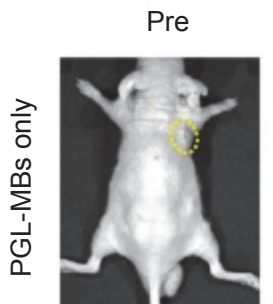

$30 \min$

$1 \mathrm{~h}$

$2 \mathrm{~h}$
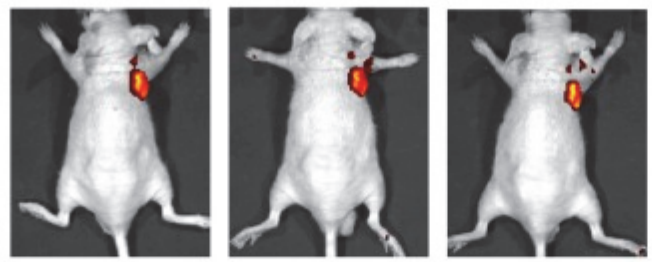

$6 \mathrm{~h}$
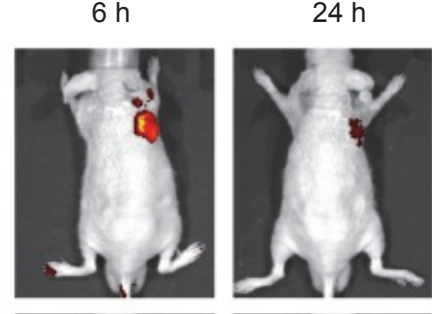

High
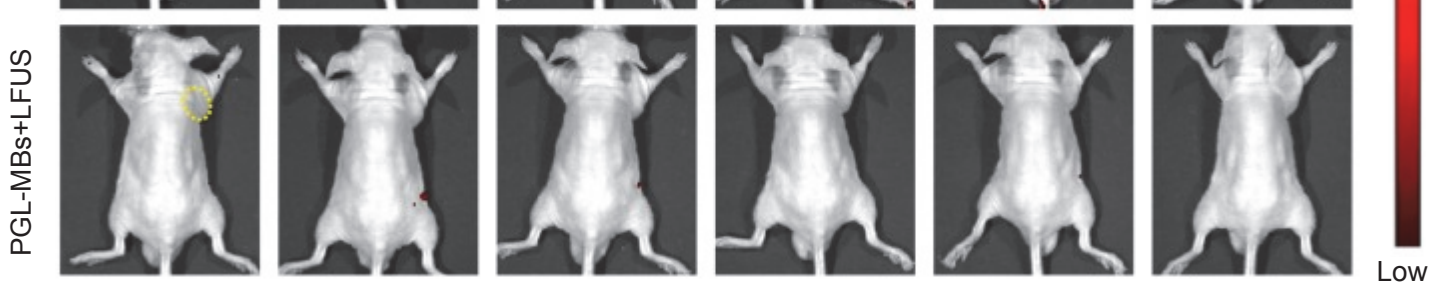

B
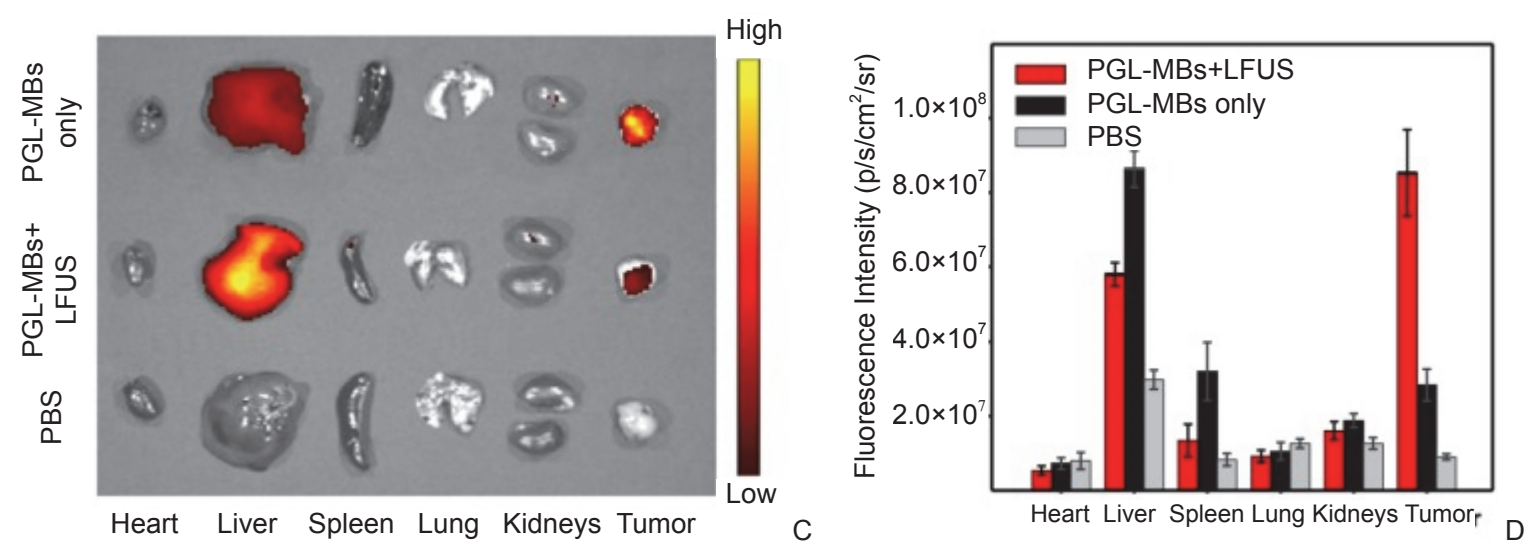

Figure 5 In vivo CEUS and fluorescence imaging in a subcutaneous PC-3 tumor model. (A) In vivo CEUS in a subcutaneous PC-3 tumor model. CEUS imaging of the tumor site before (pre) and after intravenous administration (i.v.) of PGL-MBs; (B) Fluorescence imaging in vivo at different time points after intravenous administration of PGL-MBs, with or without LFUS (400 kPa, $3 \mathrm{~min}$ ) exposure. Tumors are circled with yellow dashed lines; (C) Images of organs excised at $24 \mathrm{~h}$ after injection. Organs of tumor-bearing mice without PGL-MBs injection were excised as negative controls; (D) Quantitative analysis of fluorescence intensity for the excised organs $(n=3)$. (Reprinted with permission from reference [22])

There are few studies in the literature exploring molecular ultrasound in inflammatory bowel disease (IBD). One study in mice used mucosal addressin cellular adhesion molecule-1 (MAdCAM-1)-targeted contrast agent and VEGF- targeted contrast agents to determine inflammation changes and carcinogenesis. In mice with induced acute colitis, MAdCAM-1 expression was able to differentiate the severity of disease by detecting pixel contrast density. VEGF expression was increased in mice with tumors which correlated with IHC [28].

One study in the literature explored radiation induced colitis and found increased P-selectin expression using targeted microbubbles after radiation therapy. P-selectin and potentially other endothelial markers that are expressed in response to inflammation, such as L selection, E selectin, and ICAM-1, can be explored further to monitor inflammatory changes after radiation therapy [29]. 


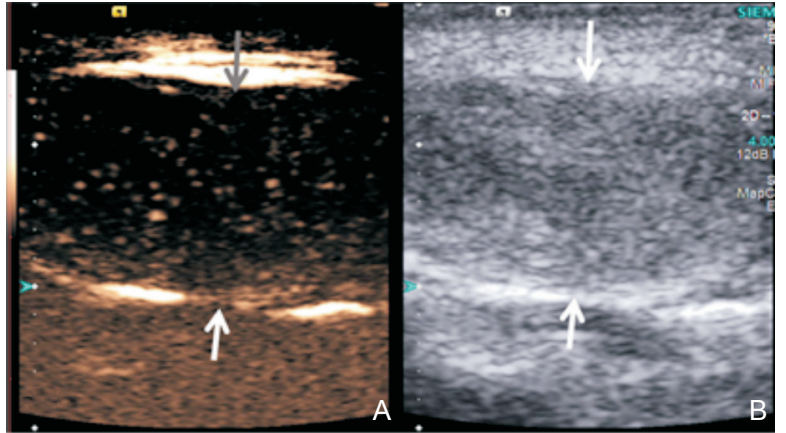

Figure 6 Malignant sentinel lymph node (arrows) in swine tumor model. (A) Contrast enhanced ultrasound image of the node with targeted microbubbles shows diffuse (42\%) enhancement. (B) B-mode ultrasound image of the heterogenous node.

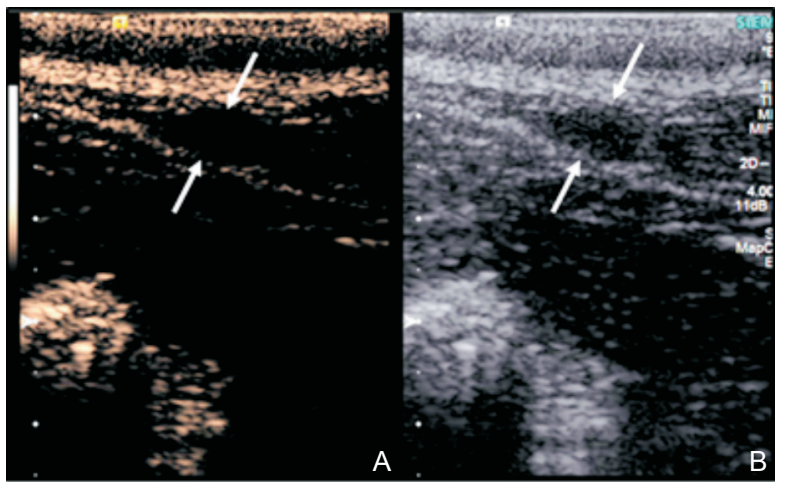

Figure 7 Benign lymph node (arrows) in swine model. (A) Contrast enhanced ultrasound image shows no retention of targeted microbubbles in the node; (B) B-mode ultrasound demonstrates homogenous lymph node, likely representing a normal lymph node.

Molecular ultrasound in colon cancer has a wide application from detecting colon cancer to assessing changes after colon cancer treatment. Future studies can utilize BR55 and other anti-angiogenesis targets to target therapy.

\section{Liver}

There are two recent studies in the literature regarding oncologic applications of molecular ultrasound in the liver. One study investigated endoglin targeted microbubbles in evaluation of angiogenesis in hepatoblastoma, which showed increased enhancement in the endoglin targeted cells [30] (Fig. 8). Another study explored VEGFR2 expression using BR55 contrast agent to evaluate therapy response in hepatocellular carcinoma and CEUS was able to detect decreased VEGFR2 expression after treatment [31]. We see similar themes as other organs in the liver with endovascular targets as the mainstay of investigation and showing promise as targets for evaluation and treatment of neoplasms.

\section{Lung}

Few studies in the literature explore CEUS in lung pathology due to the predominance of PET-CT and MRI imaging and difficulties acquiring suitable ultrasound images in the lung. However, CEUS does have some utility in small cell lung carcinoma as studied by Wang. In this study, nanobubbles were loaded with anti-progastrin releasing peptide (proGRP) antibody that target H446 cell line of small cell carcinoma and resulted in high binding affinity of the nanobubbles to the target cells [32]. Likely the future of molecular ultrasound in lung cancer will be focused on treatment delivery and future studies should focus on drug delivery as it has shown to be successful in the above study.
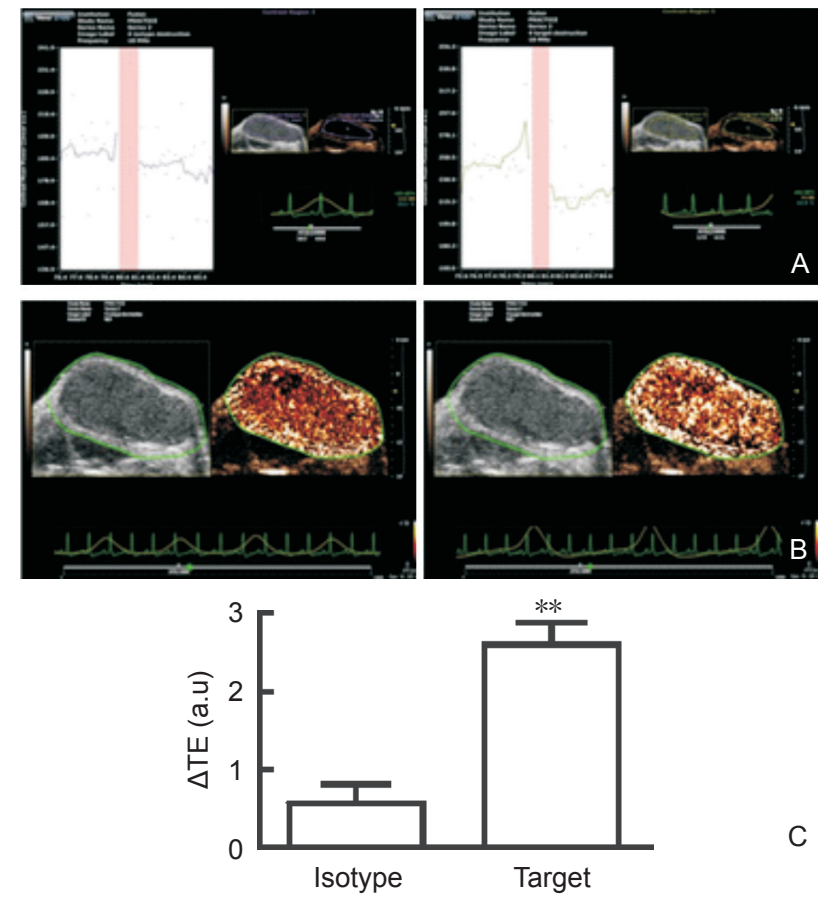

C

Figure 8 Isotype and endoglin-targeted ultrasound molecular imaging of a nude mouse with subcutaneous HB. (A) Intensity graph for the traced regions of interest prior to and after the destruction pulse (pink region). The region on the left of the column is prior to destruction, and on the right of it is after destruction. The change in intensity following the destruction pulse is an index of the amount of specific binding. The linearized signal prior to the destruction pulse represents bound and circulating microbubbles (MB) and tissue signal. The linearized signal after the destruction pulse corresponds to the MB that were still in circulation and to any residual tissue-echoes, and does not represent the binding process. The $\Delta \mathrm{TE}$ was computed by subtracting the mean intensity detected after the destructive pulse from that prior to the destructive pulse; (B) Parametric imaging. The scale bar of $\Delta T E$ is on the right corner of the map; (C) $\triangle \mathrm{TE}$ of isotype and endoglin-targeted MB. $\Delta$ TE=TEfd - TEpd. $n=4 ;{ }^{* *} P<0.001$. TE, targeted enhancement; $\Delta$ TE, differential TE; fd, following destruction; pd, prior to destruction; $\mathrm{MB}$, microbubbles. (Reprinted with permission from reference [30])

\section{Pancreas}

A recent review article on molecular imaging in pancreatic adenocarcinoma reviewed multiple contrast agents. Molecular CEUS has not been established as a diagnostic tool in pancreatic adenocarcinoma but has been established as a potential modality with increased sensitivity and specificity compared to CT. Molecular ultrasound targeting angiogenesis such as with VEGFR2 
and Thy-1 are currently being explored and have diagnostic potential [33]. Foygel initially identified Thy1 as a marker for pancreatic adenocarcinoma [33]. AbouElkacem further explored Thy-1 targeted microbubbles in detecting pancreatic adenocarcinoma by creating a novel ligand that is more specific to the target tissue and better penetrating (Thyl-scFv) [34]. Thyl appears to be the leading target for investigation currently and may prove to be a potential early diagnostic and therapeutic target.

\section{Brain}

A recent study explored molecular imaging in glioblastoma. Endoglin (CD105) targeted microbubbles were used to detect attachment numbers of endolin which correlated well with IHC. This study shows promise in using molecular ultrasound to assess angiogenesis in glioblastoma [35]. However, to date brain tumors remain a relatively unexplored area with field of molecular ultrasound.

\section{Ovarian}

The first human trial of molecular imaging agent in patients with ovarian cancer was performed with BR55 targeting KDR which matched well with IHC [8]. Due to potential instability of ligand-conjugated contrast agents, Yang et al. combined fluorescent dye, NIRF IR-780 iodide, which can target tumors, to nanobubbles. This new targeting agent successfully accumulated at female tumor cells (ovarian and breast) [36]. Gao investigated targeting CA-125 in ovarian epithelial carcinoma. Targeted nanobubbles showed tumor accumulation, more intense signal, and longer retention [37] (Fig. 9). Since BR55 is the only targeted microbubble to make it to clinical trials to date but shown limited specificity, future microbubble iterations could include drug therapy with tumor specific targets such as CA-125.

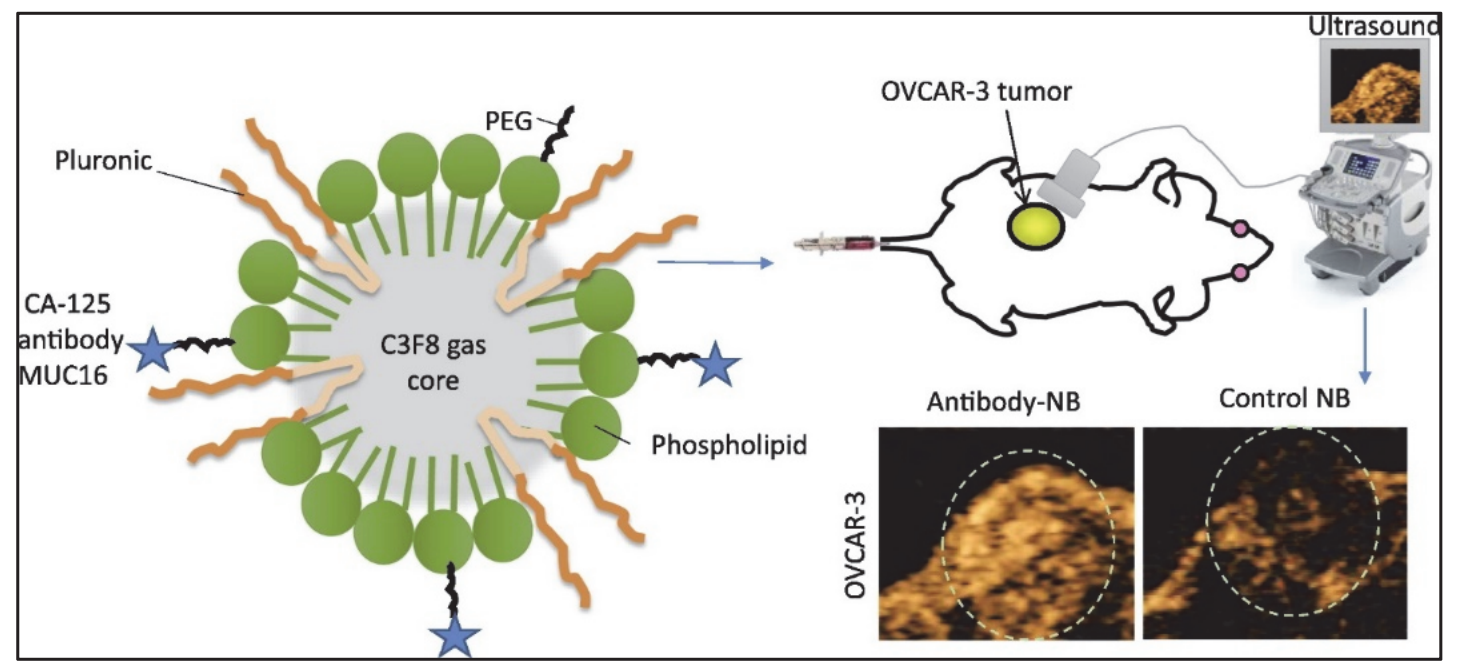

Figure 9 Ultrasound contrast agents able to target a molecular signature on the surface of cancer cells could be a promising strategy for improved cancer detection. The diagram demonstrates application of CA-125-targeted echogenic lipid and surfactant-stabilized nanobubbles (smaller than 200 nm mean hydrodynamic diameter) for targeting CA-125 positive OVCAR-3 tumors in mice. Targeted or non-targeted nanobubbles were injected into mice (via tail vein) bearing either CA-125 positive or negative flank ovarian tumors. Tumors were imaged with contrast harmonic imaging at 12 MHz, and time-intensity curves and histology were utilized to compare tumor distribution and pharmacokinetics of the contrast agents. (Reprinted with permission from reference [37])

\section{Other applications}

There are a few other applications of molecular ultrasound in the literature nonspecific to an organ system. Microbubbles targeted to tissue factor were found to be helpful in quantifying tumor burden, providing an additional method of cancer assessment and may be able to detect early, small tumors [38]. In another study, nucleolin targeted microbubbles were investigated and found to accumulate in the nucleolin cell line more than nontargeted microbubbles [39]. Zhou et al. explored Annexin V conjugated nanobubbles in assessing apoptosis. These targeted agents were unique in that they were easily able to go to tumor vasculature and accumulate at apoptotic cells, making this contrast agent promising to assess response to therapy [40].

\section{Cardiovascular Applications}

\section{Angiogenesis}

The evaluation of angiogenesis encompasses two main areas: oncology and cardiology. Although some angiogenesis studies were discussed in the 'oncology' section, many studies in the literature were more focused on generalized angiogenesis. Most cardiology applications involve evaluating angiogenesis to evaluate risk for atherosclerosis development. An earlier review of literature discussed integrin targeted and growth factor targeted contrast agents which are the current mainstay 
of investigation [41].

Two recent studies in the literature investigated integrin and growth factor targeted contrast agents including VEGF and $\alpha v \beta 3$. Wang et al. investigated VEGF targeted microbubbles and found them to be a useful method for evaluating tumor angiogenesis [42]. Daeichin et al. investigated $\alpha v \beta 3$ targeted microbubbles and found that expression was increased in atherosclerotic lesions [43].

Tumor angiogenesis is the most common application of endothelium targeted contrast agents. Three recent studies regarding integrin targeted microbubbles investigated tumor angiogenesis. Yan et al. incorporated iRGD-lipopeptides into the $\alpha \mathrm{v} \beta 3$ targeted microbubbles to increase tumor penetrability and found that this method increased signal intensity and contrast enhancement in mice with breast tumors [44]. Otani et al. created an easier to prepare lactadherin containing microbubble targeted to avb3-integrin and found that it accumulated in mice bearing tumors [45]. Hu et al. created an arginine-glycine-aspartate (RGD)-targeted microbubble to assess avb3 expression and angiogenesis. Laryngeal carcinoma (Hep-2) xenografts were used and signal was significantly increased in smaller tumors which is consistent with expected changes in angiogenesis in tumors [46] (Fig. 10).

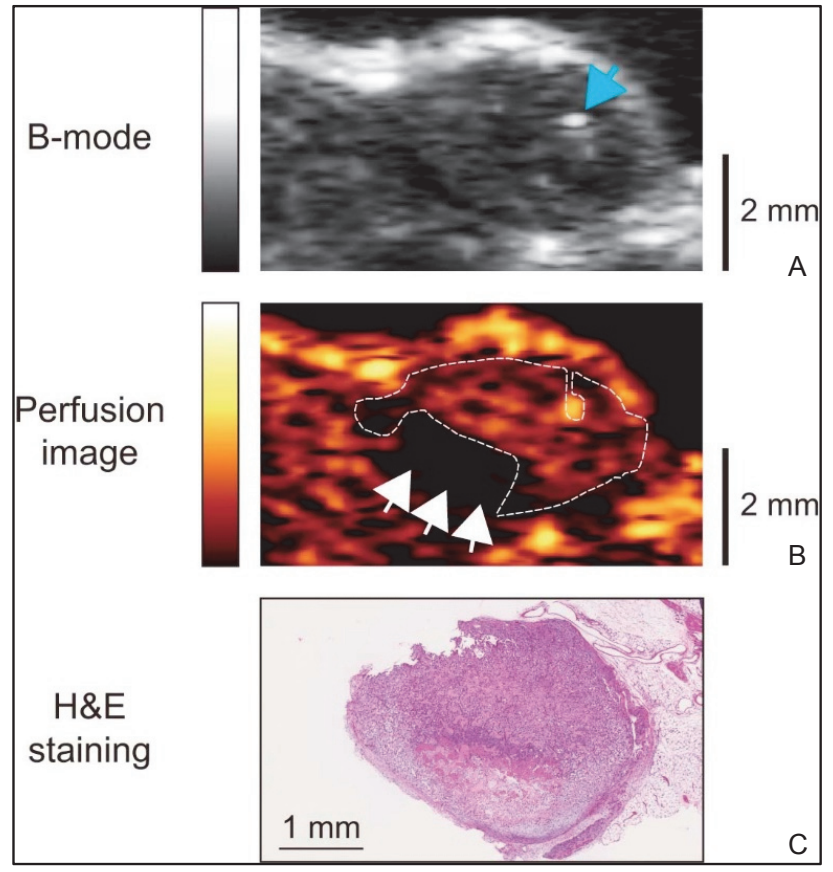

Figure 10 The region of interest (ROI) was drawn on the SK-OV-3 tumors manually so as not to encompass the areas with excessive signal intensity or perfusion defects. (Reprinted with permission from reference [45])

A few new studies explored treatment response and new contrast agents. Payen assessed the effectiveness of BR55 targeting VEGFR2 in assessing anti-angiogenesis therapy in mice with colorectal carcinoma and found that it could detect decreased tumor burden by detecting decreased VEGFR2 [47]. Since neuropilin peptides have been shown to internalize in cells, Zhang et a; evaluated the effectiveness of two neuropilin-1 targeted peptides in determining tumor angiogenesis. This study found that a new agent, CRPPR, can bind to cells that express neuropilin and can be useful in imaging angiogenesis [48].

\section{Atherosclerosis}

Most recent molecular ultrasound studies in the literature investigating atherosclerosis have focused on targets on platelets for diagnostic and therapeutic applications or assessing inflammation as a risk factor for early atherosclerosis development.

Recent studies have explored contrast agents targeted to glycoproteins on platelets to monitor atherosclerosis progression. Maier et al. studied microbubbles targeted to antibody to ligand induced binding site (LIBS) of glycoprotein IIb/IIIa of the platelet in carotid endarterectomy specimens. Signal enhancement was significantly higher after administration of the LIBSlinked microbubble [49]. Two earlier studies also explored glycoprotein targeted microbubbles. Shengcun studied microbubbles targeted to glycoprotein IIb/IIIa and found that targeting this glycoprotein specifically was associated with more vulnerable plaques, which can help prevent acute cardiac events if therapy is started early [50]. Metzger et al. found that glycoprotein-IV targeted microbubbles were diagnostic and therapeutic. After accumulation of the contrast agent was detected, high frequency ultrasound was able to interfere with and disrupt plaques which may decrease progression of atherosclerosis [51].

Other studies have linked markers of vessel inflammation to progression of atherosclerosis, especially small peptides like VCAM-1. Sun et al. used VCAM1 targeted microbubbles to assess progression of atherosclerosis and found that it was a good marker for progression since enhancement increased as plaques increased [52]. Moccetti et al. studied microbubbles targeted to multiple endothelium markers and found that VCAM1 and von Willebrand factor had the strongest signal intensity [53]. Another study assessed VCAM-1 and other small peptide endothelial markers as indicators of highrisk plaques and found that VCAM-1 showed the highest correlation with high-risk atherosclerotic disease [54].

Two relatively unique applications were investigated in the recent literature. Curaj et al. studied detection of turbulent blood flow leading to endothelial dysfunction using junctional adhesion molecule A (JAM-A) targeted microbubbles and found that CEUS was able to 
detect higher JAM-A expression at the site with more turbulent flow which can indicate a higher risk area for atherosclerotic disease [55]. Another study assessed atherosclerosis treatment response to antioxidant therapy by using P-selectin, von Willebrand factor, and glycoprotein Iba targeted microbubbles. Molecular ultrasound was able to detect response to therapy via decreased signal in all three ligands [56].

The most significant development recently has been the ability to determine atherosclerotic plaques that are at higher risk of rupture and detecting early risk of plaque development by assessing inflammation. These findings will need to be reproduced in human studies to ultimately help identify high risk individuals, provide early treatment, and prevent acute cardiac events.

\section{Inflammation}

Molecular ultrasound applications to detect inflammation are varied from vessel inflammation and injury to myocardial inflammation. Rix et al. studied avB3-integrin expression after vessel injury during endovascular interventions which can indicate injury and inflammation. This study found upregulation of the specific integrin in injured vessels and improved to baseline as vessel healed [57]. Targeting this integrin can help assess early vessel injury after interventions and help guide early treatment.

Two studies explored abdominal aorta inflammation. A study by Wang et al. successfully used P-selectin and VCAM-1 targeted microbubbles to assess inflammation in mice abdominal aorta, an early indicator for development of atherosclerosis [58]. Wu et al. compared the use of P-selectin targeted microbubbles versus dualtargeted microbubbles with the addition of PAA-sialyl Lewis-X targeting and found that $\mathrm{P}$-selectin targeted microbubbles had better adhesive efficacy under all shear stress conditions compared to the dual targeted microbubbles [59].

Myocardial inflammation caused by myocarditis and infarction was also recently studied. Leng et al. studied detection of recent myocardial ischemia using E-selectin targeted microbubbles since E-selectin is known to upregulate after myocardial ischemia. E-selectin was found to be an effective target to identify recent ischemia using echocardiography [60]. Steinl et al. evaluated detection of myocarditis using CD4 T-cell-targeted microbubbles to detect an inflammatory response and found that molecular ultrasound correlated well with the severity of inflammation [61].

Using molecular ultrasound to evaluate arterial inflammation is a promising modality to evaluate risk for atherosclerosis development. The studies exploring evaluation for myocardial ischemic changes indicate a potential non-invasive and potentially more accurate method of evaluating for ischemic cardiomyopathy in the future.

\section{Venous Thrombosis}

One recent study in the literature aimed to distinguish acute deep vein thrombosis (DVT) from chronic DVT by detecting thrombin, a marker for acute DVT. Perfluorobutane-filled (PFB-filled) microbubbles with thrombin-sensitive activatable cell-penetrating peptides (ACPPs) were created. In this in vitro study, the contrast agent was able to detect thrombin successfully while retaining $91.7 \%$ of the signal. This contrast agent could potentially be used to more accurately distinguish acute from chronic thrombus which is currently a challenge when using non-molecular ultrasound [62].

\section{Renal Applications}

Two recent studies explored molecular ultrasound applications in the kidneys. Hoyt's group investigated tissue injury due to acute kidney injury. P-selectin targeted and VCAM-1 targeted microbubbles were used to assess inflammation and P-selectin was found to be more sensitive but both molecular targets correlated with IHC [63]. Rojas et al. assessed response to antiangiogenic therapy in mice with clear-cell renal cell cancer by using VEGF targeted microbubbles and found that in $92 \%$ of treatments, molecular ultrasound was able to detect response [64] (Fig. 11). These studies indicate that molecular ultrasound may be a more accurate assessment of disease processes in the kidneys.

\section{Non-Oncologic Liver Applications}

Non-oncologic applications of molecular ultrasound imaging of the liver are focused on evaluating fibrosis and liver injury. Although other imaging molecular imaging modalities including PET have been used to stage liver fibrosis, molecular ultrasound has not been explored as extensively. Xuan et al. conjugated nanoparticles with cyclic arginine-glycine-aspartic acid (cRGD) to target avB3 which was able to distinguish stages of liver fibrosis [65].

Two recent studies in the literature evaluated ischemia-reperfusion liver injury (IRI). Qiu used ICAM-1 targeted microbubbles to determine IRI and to determine the severity of IRI [66] (Figure 12 and 13). Xie et al. targeted the same molecule in a similar study and found that targeting ICAM-1 was specific to IRI [67].

These two applications of molecular ultrasound in the liver are significant in that a less invasive method without radiation or nephrotoxic contrast agent exposure would be a preferred and more accurate modality over $\mathrm{CT}$ or MRI in the future. 

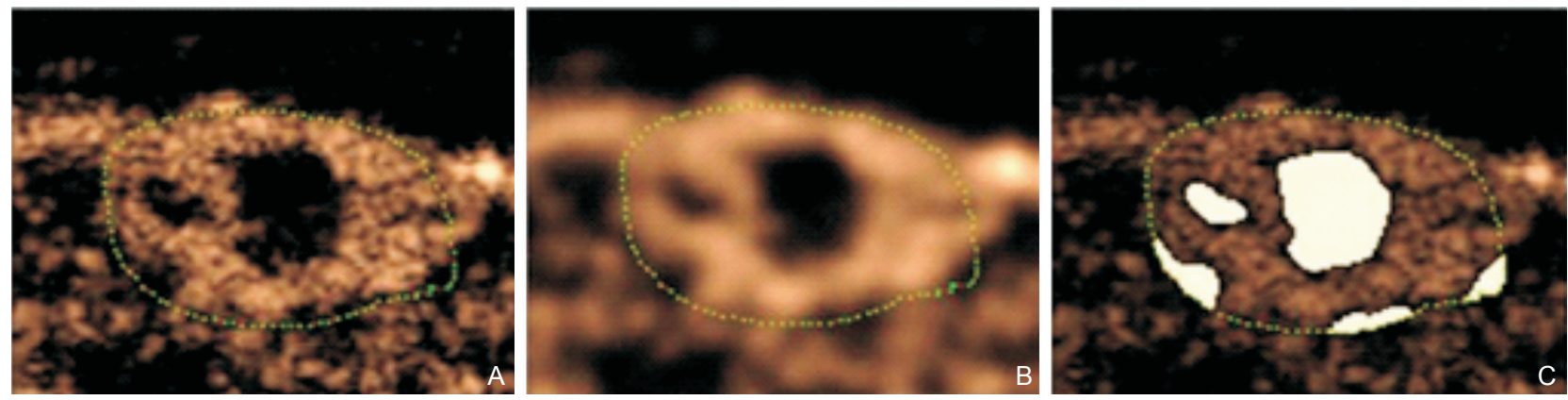

Figure 11 Molecular ultrasound imaging with VEGF targeted microbubbles detected response of anti-angiogenic therapy in mice with clear-cell cancer. Method for obtaining anechoic regions in a peak intensity image (A). The green dots outline the tumor. The image is blurred using a Gaussian filter to eliminate dark areas in speckle (B). The regions below a predetermined intensity threshold are selected as anechoic (C). Note: these are single frames in a 3D volume, so the process will be performed for each slice in the volume. (Reprinted with permission from reference [64])
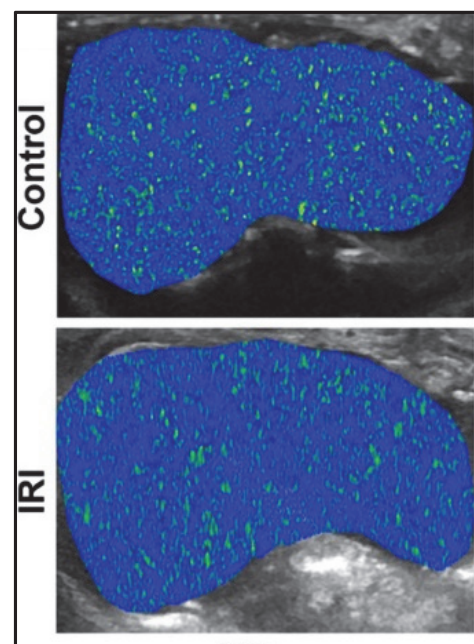

$\mathrm{MB}_{\text {Con }}$
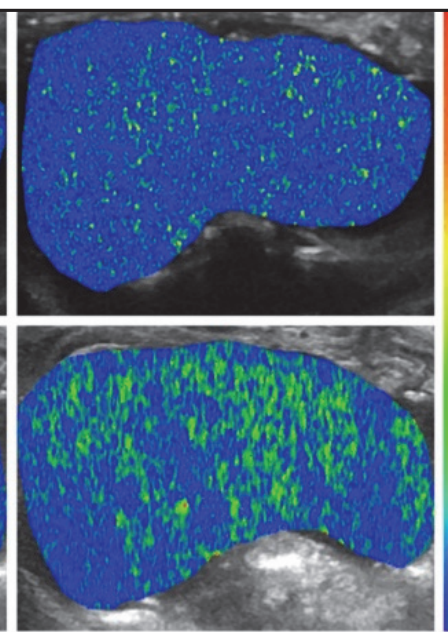

$\mathrm{MB}_{\text {ICAM-1 }}$

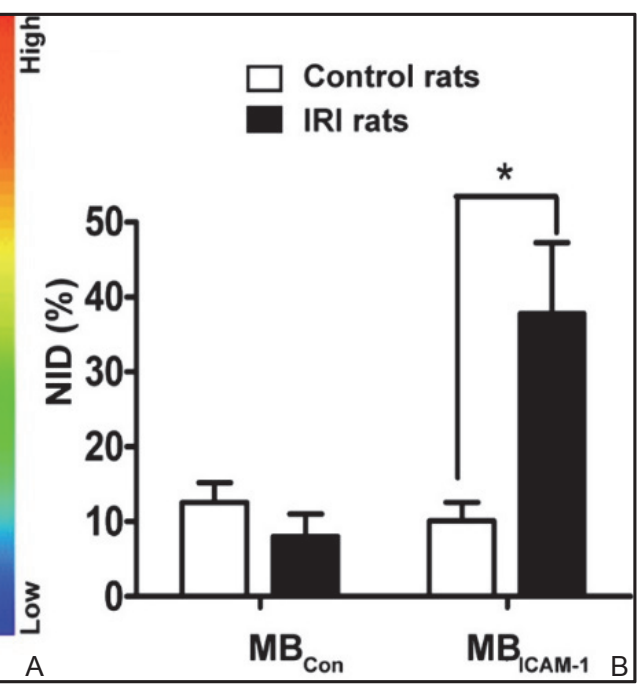

Figure 12 Representative targeted ultrasound (US) images for intracellular adhension molecule-1 (ICAM-1) and normalized intensity difference (NID) in each group. (A) Representative targeted ultrasound (US) images of ICAM-1-targeted microbubbles (MBICAM-1) and control microbubbles (MBCON) in ischemia-reperfusion injury (IRI) rats and control rats. US signal after MBICAM-1 in IRI liver was substantially higher than that in control liver and was significantly higher compared with MBCON in IRI liver and normal liver; (B) The normalized intensity differences (NIDs) were calculated as the ratio of subtraction using the destruction-replenishment method $\left(n=3,{ }^{*} P=0.048\right.$, IRI group compared with control group after MBICAM-1 administration). (Reprinted with permission from reference [66])
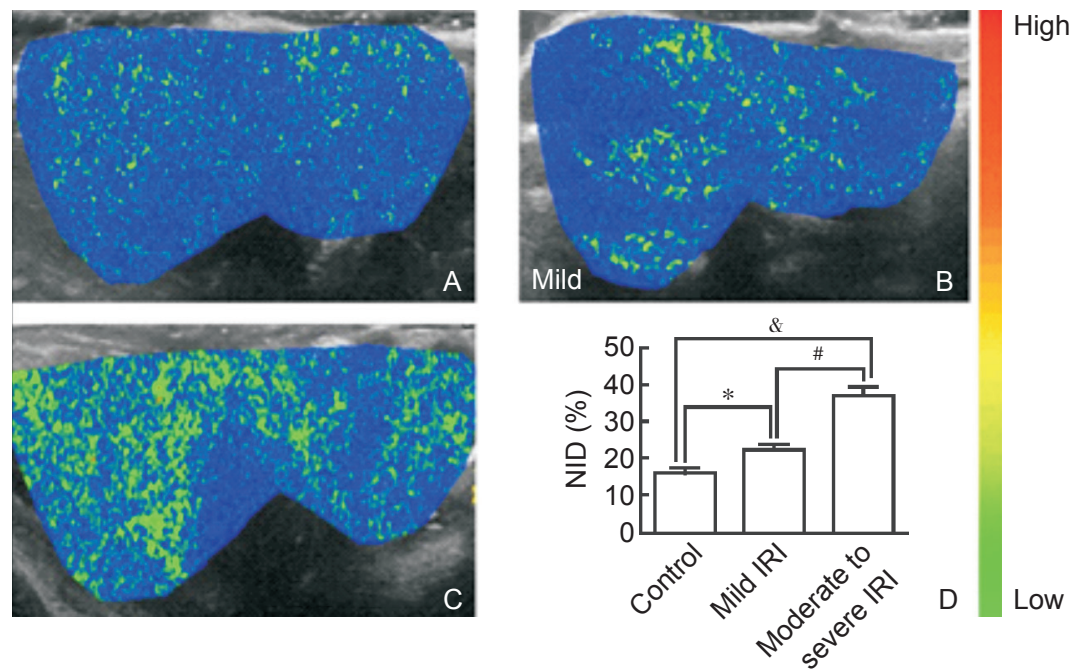

Figure 13 Representative targeted ultrasound (US) images evaluating different degrees of ischemia-reperfusion injury (IRI). Targeted US imaging signal was weakest in the control liver (A) and strongest in the moderate to severe IRI liver (C) compared with that in the mild IRI liver (B); (D) The normalized intensity difference (NID) in each group. ( ${ }^{*} P<0.001$ mild IRI group vs control group, ${ }^{\#} P=0.002$ ) moderate to severe IRI group vs mild IRI group, ${ }^{\circledR} P<0.001$ moderate to severe IRI group vs control group). (Reprinted with permission from reference [66]) 


\section{Pediatric Applications}

Much of the same applications in adults can be applied to the pediatric population. Kiessling's review outlines the preclinical studies of molecular ultrasound in different organ systems and mentions some potential applications in the pediatric population including monitoring antiangiogenic therapy, detecting arthritis, organ transplantation rejection monitoring, guiding biopsies, identifying ischemic lesions in the brain, and drug delivery [68]. However, to date few research reports have differentiated between adult and pediatric malignancies.

\section{Drug Delivery Applications}

Drug delivery is a promising application of molecular imaging and extensive research has been conducted recently in multiple fields, especially oncology.

Examples of targeted contrast agents providing gene therapy and chemotherapy for prostate cancer include nanobubbles targeted towards A10-3.2 aptamer for gene therapy [69] and chemotherapy [70]. This method was possible by ultrasound-guided contrast agent destruction at the target site to mediate drug or gene therapy delivery. Luo et al. studied a dual-targeted (folate and cRGD peptide) microbubble complex to provide chemotherapy in tumor tissues with specificity and significant accumulation [71] (Fig. 14).

In brain cancer, Chang et al. created VEGFR2targeted microbubbles for gene therapy and found that using transcranial focused ultrasound, gene therapy effectively targeted brain tumor tissue without affecting normal brain tissue when using the specific contrast agent [72]. Molecular ultrasound mediated gene therapy has also been proven successful in mice models with ovarian cancer [73].

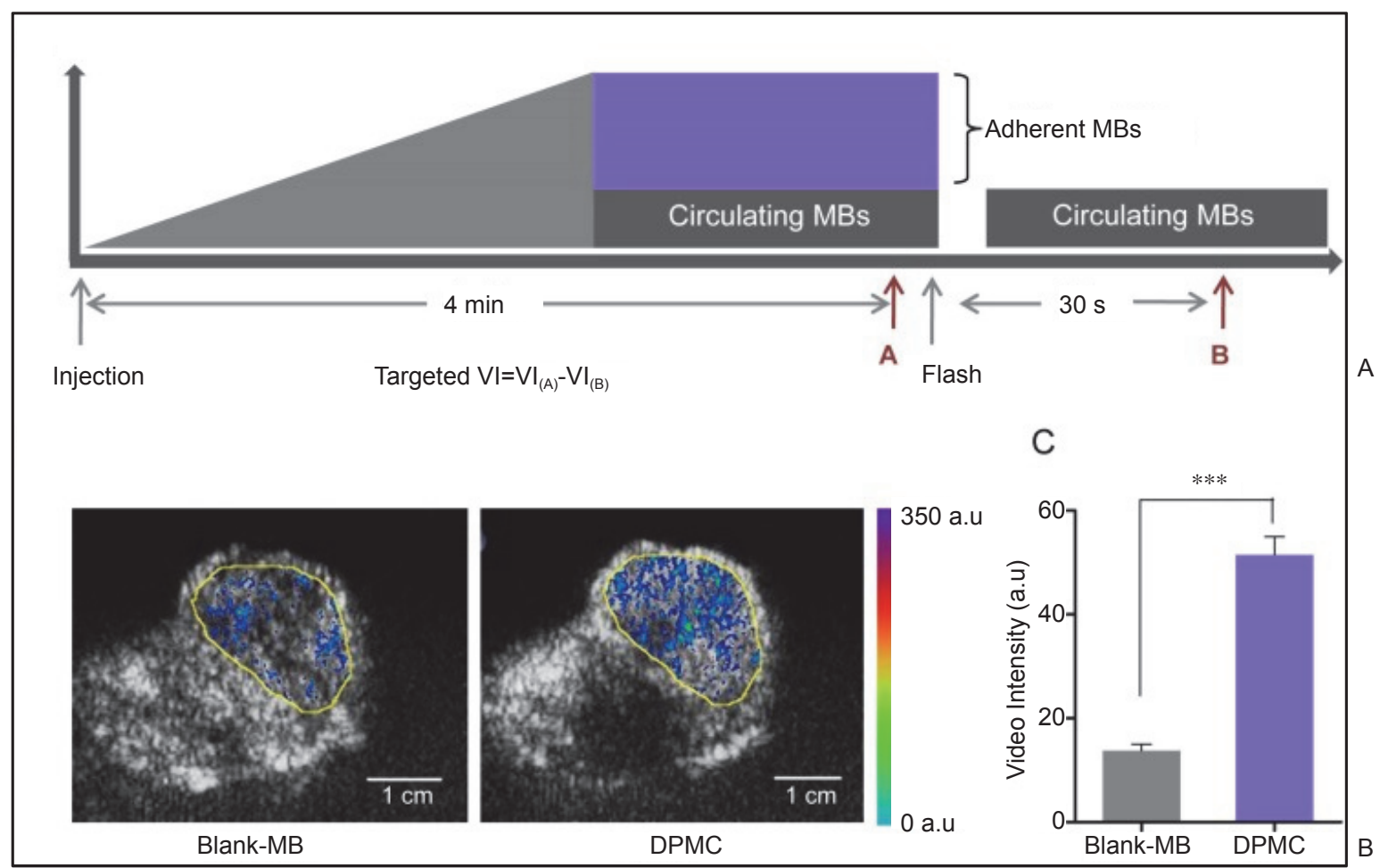

Figure 14 Schematic illustration of the image and analysis protocol (A). After monitoring for 4 min, targeted MBs were considered to adhere to the tumor site firmly. Blank MBs or DPMC were destroyed by the Flash mode of the ultrasound system. The process was monitored, starting from injection to at least $30 \mathrm{~s}$ after Flash destruction. The video intensity (VI) from adherent MBs (Targeted VI) was assessed by calculating the average pre- and post-destruction VI, and subtracting average post- from pre-destruction intensity. Ultrasound molecular images representing intensity from adherent blank MBs or DPMC displayed as color maps overlaid on B-mode images (B). Yellow line represents the region of interest (ROI). Image signals are quantitatively presented $\left({ }^{* * *} P<0.001\right)(C)$. (Reprinted with permission from reference [71])

Non-oncologic drug therapy applications include therapy for diabetic nephropathy and Crohn's disease. Yue et al. loaded microbubbles with coenzyme-Q10 and found that after treatment in rats with induced diabetic nephropathy, there was improvement in renal blood flow and recovery of renal function [74]. To provide gene therapy in Crohn's disease, MAdCAM-1 (MB-M) or
VCAM-1 (MB-V) - targeted microbubbles were used, known markers for Crohn's disease imaging and was successfully administered to the GI tract [75].

Drug therapy has great potential with the use of CEUS as a less toxic and less invasive method of administration. New applications of drug delivery mechanisms are currently being developed and CEUS 
may be the mainstay method of targeted drug therapy in the future.

\section{Conclusions}

Ultrasound molecular imaging has shown to be a romising modality particularly in the fields of oncology and cardiology. The most well-studied and currently only contrast agent to be translated to clinical trials is BR55 which is used to evaluate angiogenesis in malignancies. The most promising future applications continue to be drug delivery mechanisms and the early prediction of certain diseases such as atherosclerosis and early cancer development to guide early treatment to improve outcomes. However, to date much of the applications of ultrasound molecular imaging are in the early stages of research in animal models and need more study to gain clinical relevance.

\section{References}

[1] Deshpande N, Needles A, Willmann JK. Molecular ultrasound imaging: Current status and future directions. Clin Radiol 2010;65:567-81.

[2] Abou-Elkacem L, Bachawal S, Willmann J. Ultrasound molecular imaging: moving towards clinical translation. Eur J Radiol 2015;84:1685-93.

[3] Chong WK, Papadopoulou V, Dayton PA. Imaging with ultrasound contrast agents: current status and future. Abdom Radiol (NY) 2018;43:762-72.

[4] Güvener N, Appold L, de Lorenzi F, Golombek SK, Rizzo LY, Lammers $\mathrm{T}$, et al. Recent advances in ultrasound-based diagnosis and therapy with micro- and nanometer-sized formulations. Methods 2017; 130:4-13.

[5] Wang S, Hossack JA, Klibanov AL.Targeting of microbubbles: contrast agents for ultrasound molecular imaging. J Drug Target 2018;26:420-34.

[6] Zlitni A, Gambhir SS. Molecular imaging agents for ultrasound. Curr Opin Chem Biol 2018;45:113-20.

[7] Bzyl J, Palmowski M, Rix A, Arns S, Hyvelin JM, Pochon S, et al. The high angiogenic activity in very early breast cancer enables reliable imaging with VEGFR2-targeted microbubbles (BR55). Eur Radiol 2013;23(2):468-75.

[8] Willmann JK, Bonomo L, Testa AC, Rinaldi P, Rindi G, Valluru $\mathrm{KS}$, et al. Ultrasound molecular imaging with BR55 in patients with breast and ovarian lesions: First-in-human results. $J$ Clin Oncol 2017;35:2133-40.

[9] Bachawal SV, Jensen KC, Wilson KE, Tian L, Lutz AM, Willmann JK. Breast cancer detection by B7-H3-targeted ultrasound molecular imaging. Cancer Res 2015;75:2501-9.

[10] Wilson KE, Bachawal SV, Abou-Elkacem L, Jensen K, Machtaler $\mathrm{S}$, Tian L, et al. Spectroscopic photoacoustic molecular imaging of breast cancer using a B7-H3-targeted ICG contrast agent. Theranostics 2017;7:1463-76

[11] Wilson KE, Bachawal SV, Willmann JK. Intraoperative Resection guidance with photoacoustic and fluorescence molecular imaging using an anti-B7-H3 antibody-indocyanine green dual contrast agent. Clin Cancer Res 2018;24:3572-82.

[12] Abou-Elkacem L, Wilson KE, Johnson SM, Chowdhury SM, Bachawal S, Hackel BJ, et al. Ultrasound molecular imaging of the breast cancer neovasculature using engineered fibronectin scaffold ligands: a novel class of targeted contrast ultrasound agent. Theranostics 2016;6:1740-52.

[13] Jiang Q, Hao S, Xiao X, Yao J, Ou B, Zhao Z, et al. Production and characterization of a novel long-acting Herceptin-targeted nanobubble contrast agent specific for Her-2-positive breast cancers. Breast Cancer 2016;23:445-55.

[14] Li J, Tian Y, Shan D, Gong A, Zeng L, Ren W, et al. Neuropeptide Y Y1 receptor-mediated biodegradable photoluminescent nanobubbles as ultrasound contrast agents for targeted breast cancer imaging. Biomaterials 2017;116:106-117.

[15] Du J, Li XY, Hu H, Xu L, Yang SP, Li FH. Preparation and imaging investigation of dual-targeted C3F8-filled PLGA nanobubbles as a novel ultrasound contrast agent for breast cancer. Sci Rep $2018 ; 8: 3887$

[16] Xu L, Du J, Wan C, Zhang Y, Xie S, Li H, et al. Ultrasound molecular imaging of breast cancer in MCF-7 orthotopic mice using gold nanoshelled poly(lactic-co-glycolic acid) nanocapsules: a novel dual-targeted ultrasound contrast agent. Int J Nanomedicine 2018;13:1791-807.

[17] Wischhusen J, Wilson KE, Delcros JG, Molina-Peña R, Gibert B, Jiang S, et al. Ultrasound molecular imaging as a non-invasive companion diagnostic for netrin-1 interference therapy in breast cancer. Theranostics 2018;8:5126-42.

[18] Fischer T, Thomas A, Tardy I, Schneider M, Hünigen H, Custodis $\mathrm{P}$, et al. Vascular endothelial growth factor receptor 2-specific microbubbles for molecular ultrasound detection of prostate cancer in a rat model. Invest Radiol 2010;45:675-84.

[19] Palmowski M, Peschke P, Huppert J, Hauff P, Reinhardt M, Maurer $\mathrm{M}$, et al. Molecular ultrasound imaging of early vascular response in prostate tumors irradiated with carbon ions. Neoplasia 2009;11:856 63.

[20] Smeenge M, Tranquart F, Mannaerts CK, de Reijke TM, van de Vijver MJ, Laguna MP, et al. First-in-Human ultrasound molecular imaging with a VEGFR2-specific ultrasound molecular contrast agent (BR55) in prostate cancer: a safety and feasibility pilot study. Invest Radiol 2017;52:419-27.

[21] Fan X, Guo Y, Wang L, Xiong X, Zhu L, Fang K, et al. Diagnosis of prostate cancer using anti-PSMA aptamer A10-3.2-oriented lipid nanobubbles. Int J Nanomedicine 2016;11:3939-50.

[22] You Y, Liang X, Yin T, Chen M, Qiu C, Gao C, et al. Porphyringrafted lipid microbubbles for the enhanced efficacy of photodynamic therapy in prostate cancer through ultrasound-controlled in situ accumulation. Theranostics 2018;8:1665-77.

[23] Nam K, Stanczak M, Forsberg F, Liu JB, Eisenbrey JR, Solomides $\mathrm{CC}$, et al. Sentinel lymph node characterization with a dual-targeted molecular ultrasound contrast agent. Mol Imaging Biol 2018;20:2219

[24] Hackl C, Schacherer D, Anders M, Wiedemann LM, Mohr A, Schlitt $\mathrm{HJ}$, et al. Improved detection of preclinical colorectal liver metastases by high resolution ultrasound including molecular ultrasound imaging using the targeted contrast agent BR55. Ultraschall Med 2016;37:290-6

[25] Eschbach RS, Clevert DA, Hirner-Eppeneder H, Ingrisch M, Moser M, Schuster J, et al. Contrast-Enhanced ultrasound with VEGFR2 targeted microbubbles for monitoring regorafenib therapy effects in experimental colorectal adenocarcinomas in rats with DCE-MRI and immunohistochemical validation. PLoS One 2017;12:e169323.

[26] Zhou J, Wang H, Zhang H, Lutz AM, Tian L, Hristov D, et al. VEGFR2-targeted three-dimensional ultrasound imaging can predict responses to antiangiogenic therapy in preclinical models of colon cancer. Cancer Res 2016;76:4081-9. 
[27] Wang H, Kaneko OF, Tian L, Hristov D, Willmann JK. Threedimensional ultrasound molecular imaging of angiogenesis in colon cancer using a clinical matrix array ultrasound transducer. Invest Radiol 2015;50:322-9.

[28] Brückner M, Heidemann J, Nowacki TM, Cordes F, Stypmann J, Lenz P, et al. Detection and characterization of murine colitis and carcinogenesis by molecularly targeted contrast-enhanced ultrasound. World J Gastroenterol 2017;23:2899-911.

[29] El Kaffas A, Smith K, Pradhan P, Machtaler S, Wang H, von Eyben R, et al. Molecular contrast-enhanced ultrasound imaging of radiationinduced P-selectin expression in healthy mice colon. Int J Radiat Oncol Biol Phys 2017;97:581-5.

[30] Shan R, Wang B, Wang A, Sun Z, Dong F, Liu J, et al. Endoglintargeted contrast-enhanced ultrasound imaging in hepatoblastoma xenografts. Oncol Lett 2018;16:3784-90.

[31] Baron Toaldo M, Salvatore V, Marinelli S, Palamà C, Milazzo M, Croci L, et al. Use of VEGFR-2 targeted ultrasound contrast agent for the early evaluation of response to sorafenib in a mouse model of hepatocellular carcinoma. Mol Imaging Biol 2015;17:29-37.

[32] Wang JP, Zhou XL, Yan JP, Zheng RQ, Wang W. Nanobubbles as ultrasound contrast agent for facilitating small cell lung cancer imaging. Oncotarget 2017;8:78153-62.

[33] Tummers WS, Willmann JK, Bonsing BA, Vahrmeijer AL, Gambhir SS, Swijnenburg RJ. Advances in diagnostic and intraoperative molecular imaging of pancreatic cancer. Pancreas 2018;47:675-89.

[34] Abou-Elkacem L, Wang H, Chowdhury SM, Kimura RH, Bachawal SV, Gambhir SS, et al. Thy1-targeted microbubbles for ultrasound molecular imaging of pancreatic ductal adenocarcinoma. Clin Cancer Res 2018;24:1574-85.

[35] Liu C, Yan F, Xu Y, Zheng H, Sun L. In vivo molecular ultrasound assessment of glioblastoma neovasculature with Endoglin-targeted microbubbles. Contrast Media Mol Imaging 2018;2018:8425495.

[36] Yang H, Zhou T, Cai W, Yi X, Liu X, Wang Y, et al. Novel dual-mode nanobubbles as potential targeted contrast agents for female tumors exploration. Tumour Biol 2016;37:14153-63.

[37] Gao Y, Hernandez C, Yuan HX, Lilly J, Kota P, Zhou H, et al. Ultrasound molecular imaging of ovarian cancer with CA-125 targeted nanobubble contrast agents. Nanomedicine 2017;13:215968.

[38] Sciallero C, Daglio E, Trucco A. In vivo quantification of ultrasound targeted microbubbles to enhance cancer assessment. Contrast Media Mol Imaging 2016;11:313-18.

[39] Zhang H, Ingham ES, Gagnon MK, Mahakian LM, Liu J, Foiret $\mathrm{JL}$, et al. In vitro characterization and in vivo ultrasound molecular imaging of nucleolin-targeted microbubbles. Biomaterials 2017; 118:63-73.

[40] Zhou T, Cai W, Yang H, Zhang H, Hao M, Yuan L, et al. Annexin V conjugated nanobubbles: A novel ultrasound contrast agent for in vivo assessment of the apoptotic response in cancer therapy. $J$ Control Release 2018;276:113-24.

[41] Eisenbrey JR, Forsberg F. Contrast-enhanced ultrasound for molecular imaging of angiogenesis. Eur J Nucl Med Mol Imaging 2010;37 Suppl 1:S138-46.

[42] Wang J, Qin B, Chen X, Wagner WR, Villanueva FS. Ultrasound molecular imaging of angiogenesis using vascular endothelial growth factor-conjugated microbubbles. Mol Pharm 2017; 14:781-90.

[43] Daeichin V, Kooiman K, Skachkov I, Bosch JG, Theelen TL, Steiger $\mathrm{K}$, et al. Quantification of endothelial $\alpha v \beta 3$ expression with highfrequency ultrasound and targeted microbubbles: in vitro and in vivo studies. Ultrasound Med Biol 2016;42:2283-93.

[44] Yan F, Xu X, Chen Y, Deng Z, Liu H, Xu J, et al. A LipopeptideBased $\alpha$ v $\beta$ Integrin-targeted ultrasound contrast agent for molecular imaging of tumor angiogenesis. Ultrasound Med Biol. 2015;41:276573.

[45] Otani K, Nishimura H, Kamiya A, Harada-ShibaM. Simplified preparation of $\alpha v \beta 3$ Integrin-targeted microbubbles based on a clinically available ultrasound contrast agent: validation in a tumorbearing mouse model. Ultrasound Med Biol 2018;44:1063-73.

[46] Hu Q, Wang XY, Kang LK, Wei HM, Xu CM, Wang T, et al. RGDtargeted ultrasound contrast agent for longitudinal assessment of Hep-2 tumor angiogenesis in vivo. PLoS One 2016;11:e0149075.

[47] Payen T, Dizeux A, Baldini C, Le Guillou-Buffello D, Lamuraglia M, Comperat E, et al. VEGFR2-targeted contrast-enhanced ultrasound to distinguish between two anti-angiogenic treatments. Ultrasound Med Biol 2015;41:2202-11.

[48] Zhang H, Tam S, Ingham ES, Mahakian LM, Lai CY, Tumbale SK, et al. Ultrasound molecular imaging of tumor angiogenesis with a neuropilin-1-targeted microbubble. Biomaterials 2015;56:104-13.

[49] Maier A, Plaza-Heck P, Meixner F, Guenther F, Kaufmann BA, Kramer M, et al. A molecular intravascular ultrasound contrast agent allows detection of activated platelets on the surface of symptomatic human plaques. Atherosclerosis 2017;267:68-77.

[50] Guo S, Shen S, Wang J, Wang H, Li M, Liu Y, et al. Detection of high-risk atherosclerotic plaques with ultrasound molecular imaging of glycoprotein IIb/IIIa receptor on activated platelets. Theranostics 2015;5:418-30.

[51] Metzger K, Vogel S, Chatterjee M, Borst O, Seizer P, Schönberger T, et al. High-frequency ultrasound-guided disruption of glycoprotein VI-targeted microbubbles targets atheroprogressison in mice. Biomaterials 2015;36:80-9.

[52] Sun R, Tian J, Zhang J, Wang L, Guo J, Liu Y. Monitoring inflammation injuries in the progression of atherosclerosis with contrast enhanced ultrasound molecular imaging. Plos One 2017;12:e0186155.

[53] Moccetti F, Weinkauf CC, Davidson BP, Belcik JT, Marinelli ER, Unger E, et al. Ultrasound molecular imaging of atherosclerosis using small-peptide targeting ligands against endothelial markers of inflammation and oxidative stress. Ultrasound Med Biol 2018;44:1155-63

[54] Weinkauf CC, Concha-Moore K, Lindner JR, Marinelli ER, Hadinger $\mathrm{KP}$, Bhattacharjee S, et al. Endothelial vascular cell adhesion molecule 1 is a marker for high-risk carotid plaques and target for ultrasound molecular imaging. J Vasc Surg 2018;68:105S-13S.

[55] Curaj A, Wu Z, Rix A, Gresch O, Sternkopf M, Alampour-Rajabi S et al. Molecular ultrasound imaging of junctional adhesion molecule a depicts acute alterations in blood flow and early endothelial dysregulation. Arterioscler Thromb Vasc Biol 2018;38:40-8.

[56] Atkinson T, Packwood W, Xie A, Liang S, Qi Y, Ruggeri Z, et al. Assessment of novel antioxidant therapy in atherosclerosis by contrast ultrasound molecular imaging. J Am Soc Echocardiogr. 2018;31:1252-9

[57] Rix A, Fokong S, Heringer S, Pjontek R, Kabelitz L, Theek B, et al. Molecular ultrasound imaging of $\alpha \mathrm{v} \beta 3$-Integrin expression in carotid arteries of pigs after vessel injury. Invest Radiol 2016;51:76775 .

[58] Wang S, Unnikrishnan S, Herbst EB, Klibanov AL, Mauldin FW Jr, Hossack JA. Ultrasound molecular imaging of inflammation in mouse abdominal aorta. Invest Radiol 2017;52:499-506.

[59] Wu W, Feng X, Yuan Y, Liu Y, Li M, Bin J, et al. Comparison of magnetic microbubbles and dual-modified microbubbles targeted to P-selectin for imaging of acute endothelial inflammation in the abdominal aorta. Mol Imaging Biol 2017;19:183-93.

[60] Leng X, Wang J, Carson A, Chen X, Fu H, Ottoboni S, et al. Ultrasound detection of myocardial ischemic memory using an 
E-selectin targeting peptide amenable to human application. Mol Imaging 2014;13:1-9.

[61] Steinl DC, Xu L, Khanicheh E, Ellertsdottir E, Ochoa-Espinosa A, Mitterhuber $\mathrm{M}$, et al. Noninvasive contrast-enhanced ultrasound molecular imaging detects myocardial inflammatory response in autoimmune myocarditis. Circ Cardiovasc Imaging 2016;9. pii: e004720.

[62] Lux J, Vezeridis AM, Hoyt K, Adams SR, Armstrong AM, Sirsi SR, et al. Thrombin-activatable microbubbles as potential ultrasound contrast agents for the detection of acute thrombosis. ACS Appl Mater Interfaces 2017;9:37587-96.

[63] Hoyt K, Warram JM, Wang D, Ratnayaka S, Traylor A, Agarwal A. Molecular ultrasound imaging of tissue inflammation using an animal model of acute kidney injury. Mol Imaging Biol 2015;17:786-92.

[64] Rojas JD, Lin F, Chiang YC, Chytil A, Chong DC, Bautch VL, et al. Ultrasound molecular imaging of VEGFR-2 in clear-cell renal cell carcinoma tracks disease response to antiangiogenic and notchinhibition therapy. Theranostics 2018;8:141-55.

[65] Xuan J, Chen Y, Zhu L, Guo Y, Deng L, Zheng Y, et al. Ultrasound molecular imaging with cRGD-PLGA-PFOB nanoparticles for liver fibrosis staging in a rat model. Oncotarget 2017;8:108676-91.

[66] Qiu C, Yin T, Zhang Y, Lian Y, You Y, Wang K, et al. Ultrasound imaging based on molecular targeting for quantitative evaluation of hepatic ischemia-reperfusion injury. Am J Transplant 2017;17:308797.

[67] Xie F, Li ZP, Wang HW, Fei X, Jiao ZY, Tang WB, et al. Evaluation of liver ischemia-reperfusion injury in rabbits using a nanoscale ultrasound contrast agent targeting ICAM-1. PLoS One 2016;11:e153805.
[68] Kiessling I, Bzyl J, Kiessling F. Molecular ultrasound imaging and its potential for paediatric radiology. Pediatr Radiol 2011;41:176-84.

[69] Wu M, Zhao H, Guo L, Wang Y, Song J, Zhao X, et al. Ultrasoundmediated nanobubble destruction (UMND) facilitates the delivery of A10-3.2 aptamer targeted and siRNA-loaded cationic nanobubbles for therapy of prostate cancer. Drug Deliv 2018;25:226-40.

[70] Wu M, Wang Y, Wang Y, Zhang M, Luo Y, Tang J, et al. Paclitaxelloaded and A10-3.2 aptamer-targeted poly(lactide-co-glycolic acid) nanobubbles for ultrasound imaging and therapy of prostate cancer. Int J Nanomedicine 2017;12:5313-30.

[71] Luo W, Wen G, Yang L, Tang J, Wang J, Wang J, et al. Dual-targeted and $\mathrm{pH}$-sensitive Doxorubicin Prodrug-Microbubble Complex with Ultrasound for Tumor Treatment. Theranostics 2017;7:452-65.

[72] Chang EL, Ting CY, Hsu PH, Lin YC, Liao EC, Huang CY, et al. Angiogenesis-targeting microbubbles combined with ultrasoundmediated gene therapy in brain tumors. $J$ Control Release 2017;255:164-75.

[73] Yang C, Li B, Yu J, Yang F, Cai K, Chen Z. Ultrasound microbubbles mediated miR-let-7b delivery into CD133+ ovarian cancer stem cells. Biosci Rep 2018;38. pii: BSR20180922.

[74] Yue T, Xu HL, Chen PP, Zheng L, Huang Q, Sheng WS, et al. Combination of coenzyme Q10-loaded liposomes with ultrasound targeted microbubbles destruction (UTMD) for early theranostics of diabetic nephropathy. Int J Pharm 2017;528:664-74.

[75] Tlaxca JL, Rychak JJ, Ernst PB, Konkalmatt PR, Shevchenko TI, Pizarro TT, et al. Ultrasound-based molecular imaging and specific gene delivery to mesenteric vasculature by endothelial adhesion molecule targeted microbubbles in a mouse model of Crohn's disease. J Control Release 2013;165:216-25. 